\title{
Impact of Star Connection Layouts on the Control of Multiphase Induction Motor Drives Under Open-Phase Fault
}

\author{
Giacomo Sala, Member, IEEE, Michele Mengoni, Member, IEEE, Gabriele Rizzoli, \\ Michele Degano, Member, IEEE, Luca Zarri, Senior Member, IEEE, and Angelo Tani.
}

\begin{abstract}
This paper presents a post-fault control algorithm that minimizes the stator Joule losses in multiphase induction machines under an open-phase fault and for different star connection layouts. The key novelty is that the algorithm can be applied to any configuration of a multi $n$-phase machine, independently of the connection of the neutral points. The latter is analytically derived and is based on the space vector representation of the machine model. Also, it is shown that a low number of neutral points helps to reduce the winding losses in case of an open-phase fault but requires additional control regulators and computational efforts. The theory is applied to an asymmetrical quadruple three-phase induction machine, which is configured to represent five different motor layouts. Finally, experimental results are presented to validate the control algorithm. The optimal solution that is given in the paper can be employed for the control of symmetrical or asymmetrical multiphase machines with different star connection layouts and in any open-phase post-fault operation.
\end{abstract}

Index Terms-Circuit faults, post-fault control, induction motors, multiphase machines, power system reliability, variable speed drives.

\section{INTRODUCTION}

W HEN reliability is an essential feature, a standard approach to enhance the fault tolerance is the redundancy of the critical systems. In the field of electric drives, the use of multiphase machines is considered as one of the most promising fault-tolerant technologies [1], [2]. An initial classification of multiphase drives can be done considering the layout of the stator phases. Therefore, multiphase drives are commonly distinguished in multiphase, multi single-phase and multi three-phase ones [3].

In a multiphase drive where a single converter feeds all the phases connected to a single neutral point a variety of critical faults may affect the system and lead to the complete failure of the drive (dc-bus failure, power converter fault,

Manuscript received May 14, 2020; revised July 3, 2020; accepted September 8,2020 .

G. Sala, M. Mengoni, G. Rizzoli, L. Zarri and A. Tani are with the Department of Electrical and Electronic Engineering, University of Bologna, Bologna (e-mail: g.sala@unibo.it, michele.mengoni@unibo.it, gabriele.rizzoli@unibo.it, luca.zarri@unibo.it angelo.tani@unibo.it).

M. Degano is with the PEMC Group, Department of Electrical and Electronic Engineering, The University of Nottingham, NG7 2RD, UK (email: michele.degano@nottingham.ac.uk). M. Degano is also with the Key Laboratory of More Electric Aircraft Technology of Zhejiang Province, PEMC Group, University of Nottingham Ningbo Campus, UNNC, China. short circuits in the winding, etc.). To avoid the failure of the system in these scenarios, one of the most reliable layout is the multi single-phase, where independent converters feed galvanically separated phases. However, this solution may be complicated, expensive and with reduced efficiency [4]. The multi three-phase layout is an intermediate solution, where each three-phase winding is star-connected and can be fed by an independent inverter. Multi $n$-phase layouts can also be adopted, but the three-phase one is favorite because of the maturity of the related power-electronics technology. For example, Fig. 1 illustrates the multi three-phase layout of the twelve-phase machine considered for the validation of the algorithm proposed in this work.

Multiphase drives are redundant by definition: when a phase is open, the other ones can compensate for the missing power, and reduce the performance de-rating. However, the optimal post-fault strategy is challenging to generalize, because it depends on the machine topology and the control algorithm. Some research lines dealt with methodologies for the definition of optimum current references to obtain a better torque response. These methods have been developed for machines with different layouts and numbers of phases by using genetic algorithms [5], numerical computations based on analytical formulations [6], and categorizing the angles between the adjacent healthy phases [7]. The fault-tolerant control systems have also been integrated with diagnostic algorithms that take into account for the converter layout [8]. Also, as confirmed by recent studies, the performance of conventional control schemes for multiphase drives significantly deteriorates under open-phase faults and requires a change of the architecture toward a fault-tolerant scheme [9]. This phenomenon is well

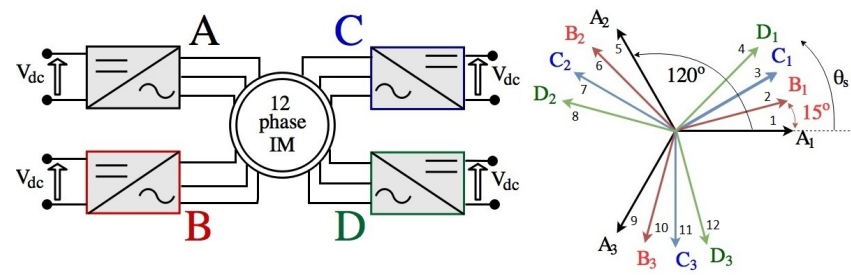

Fig. 1. Conceptual scheme of the analyzed drive in its quadruple three-phase layout (left) and magnetic axes of the twelve phase induction machine (right). The axes of each three-phase subsystem are equally colored, and are shifted by 15 electrical degrees with respect to each other. 
described also in [10], where a self-healing control scheme is proposed. In that paper, the architecture of the control scheme does not change when a fault happens, and no fault detection is required thanks to an Extended Phase Locked Loop (EPLL). The EPLL is embedded with an automatic adjustment of the reference currents in the harmonic subspace limited by a minimum loss coefficient.

This paper investigates how the star connection layout affects the performance of multi $n$-phase induction machines under open-phase fault conditions. Some post-fault algorithms have already been proposed for induction machines with a single star and an odd number of phases [11]. Later, they have been extended to dual three-phase machines with single or double isolated neutral points [12], [13]. Various fault-tolerant algorithms have been compared, showing how to obtain the maximum output torque under open-phase fault scenarios. However, at the current state of the art, several post-fault algorithms require the numerical solution of an optimization problem with convergence methods that avoid local maximums and guarantee a global optimum solution [14]. These methods can be applied to any electrical machine. They have also been extended to delta-connected layouts [15]-[17] and to customized inverters or peculiar winding layouts, e.g., to enhance the performance or the fault-tolerant capabilities [18], [19]. If the post-fault control aims to minimize the stator copper losses, it is also possible to find the optimal analytical solution in closed form, as demonstrated for particular configurations of the windings [11], [20], [21] and converter architectures [22], [23].

One of the aims of the current research is to develop faulttolerant algorithms that are as general as possible. Furthermore, the analysis of the stator Joule losses and their distribution in the machine winding has a significant impact on the post-fault thermal stress [24]-[27], making its minimization a priority for post-fault algorithms of electrical machines.

With reference to these topics, the main contributions of the present paper are listed hereafter.

(i) The work defines a generalized method for post-fault control of multiphase machines in case of open-phase faults, which can be analytically derived and easily adapted to different machine layouts. For the sake of generality, the stator phases are arranged into a set of $n$ phase windings, where the neutral points of each winding can be connected to or insulated from the neutral points of the other windings, depending on the machine layout. To the best of the authors' knowledge, such a method is not available in the literature.

(ii) In case of an open-phase fault, it is necessary to find the optimal set-points of the machine currents, to mitigate the effect of the fault. It turns out that the system of equations that solves this problem depends on the specific machine layout and, in some cases, is even undetermined. To overcome this difficulty, the developed algorithm minimizes the Joule losses and finds an explicit solution by using the pseudo-inverse operator and the Vector Space Decomposition (VSD) approach [28].

Finally, experimental results are presented to validate the post-fault control and to provide an in-depth comparison of the developed techniques for the single-, double-, and quadruplestar layouts of a twelve-phase machine.

\section{Machine Equations in HeAlthy And FAulty CONDITIONS}

The machine model is developed decomposing the machine variables in multiple vector sub-spaces or planes. Consequently, the equations written in terms of Space Vectors (SVs) can be used to evaluate the electromechanical behavior of the machine and develop a suitable control algorithm.

\section{A. Space Vector Representation}

VSD is widely used for the analysis of multiphase machines and allows representing any set of electrical quantities $y_{1}, y_{2}, \ldots, y_{m}$ in terms of their respective $\mathrm{SVs} \bar{y}_{1}, \bar{y}_{2}, \ldots, \bar{y}_{m}$ defined as follows:

$$
\bar{y}_{\rho}=\frac{2}{m} \sum_{k=1,2, \ldots}^{m} y_{k} e^{j \rho \varphi_{k}}
$$

where $\varphi_{k}$ is the angular position of the magnetic axis of the $k t h$ phase. In a multi $n$-phase machine with $m / n n$-phase sub-windings arranged symmetrically, the angle $\varphi_{k}$ can be calculated as follows:

$$
\varphi_{k}=\bmod \left(k-1, \frac{m}{n}\right) \Delta \varphi+\left\lfloor(k-1) /\left(\frac{m}{n}\right)\right\rfloor \frac{2 \pi}{n}
$$

where $\bmod$ is the modulo operation that finds the remainder after $(k-1)$ is divided by $\left.\left.\frac{m}{n},\right\rfloor\right\rfloor$ is the floor operation that rounds its argument down to an integer, and $\Delta \varphi$ is the angular shift among neighboring sub-windings. Also, the first phase $(k=1)$ is assumed aligned with the stator reference frame $\left(\varphi_{1}=0\right)$.

In case of distributed windings with $m$ phases, the angular shift $\Delta \varphi$ of the sub-windings is $\frac{2 \pi}{m}$ for a symmetrical layout, and $\frac{\pi}{m}$ for an asymmetrical layout (as illustrated in Fig. 1).

If the machine features an odd number of phases, the inverse transformation of (1) can be simplified as follows:

$$
y_{k}=\frac{\bar{y}_{m}}{2} e^{-j m \varphi_{k}}+\sum_{\rho=1,3,5, \ldots}^{m-2} \Re\left\{\bar{y}_{\rho} e^{-j \rho \varphi_{k}}\right\}
$$

where $\Re$ is the real operator.

Conversely, for a machine with an even number of phases, the inverse transformation of (1) becomes:

$$
y_{k}=\sum_{\rho=1,3,5 \ldots}^{m-1} \Re\left\{\bar{y}_{\rho} e^{-j \rho \varphi_{k}}\right\} .
$$

Equations (1) - (4) can be used to develop models and control algorithms for multiphase machines, independently of their star-connection layouts. 


\section{B. Model of a Multiphase Induction Machine}

The stator and rotor equations describing a multiphase induction machine are written in the stator and rotor reference frames, respectively, in terms of SVs as follows:

$$
\begin{gathered}
\bar{v}_{S, \rho}=R_{S} \bar{i}_{S, \rho}+\frac{d \bar{\phi}_{S, \rho}}{d t} \\
0=R_{R, \rho} \bar{i}_{R, \rho}+\frac{d \bar{\phi}_{R, \rho}}{d t}
\end{gathered}
$$

where $R_{S}$ and $R_{R, \rho}$ are the stator phase resistance and equivalent rotor resistance in the $\rho$ th subspace. Neglecting the iron saturation, the slotting effect, and the high-order field harmonics with $\rho>1$, the stator and rotor flux vectors are as follows:

$$
\begin{gathered}
\bar{\phi}_{S, 1}=L_{S, 1} \bar{i}_{S, 1}+M_{1} \bar{i}_{R, 1} e^{j \theta_{m}} \\
\bar{\phi}_{R, 1}=L_{R, 1} \bar{i}_{R, 1}+M_{1} \bar{i}_{S, 1} e^{-j \theta_{m}} \\
\bar{\phi}_{S, \rho}=l_{S, \rho} \bar{i}_{S, \rho} \quad \rho=3,5, \ldots \\
\bar{\phi}_{R, \rho}=l_{R, \rho} \bar{i}_{R, \rho} \quad \rho=3,5, \ldots
\end{gathered}
$$

where $\theta_{m}$ is the angular displacement between the stator and rotor reference frames in electrical radians, $L_{S, 1}, L_{R, 1}$ and $M_{1}$ are the stator, rotor and mutual inductances of the fundamental harmonic, while $l_{S, \rho}$ and $l_{R, \rho}$ are the equivalent stator and rotor leakage inductances in the $\rho t h$ subspace. Finally, under the assumption that the high-order spatial components of the magnetic field are negligible, the electromagnetic torque can be written as follows:

$$
T=\frac{m}{2} p M_{1} \Re\left\{j \bar{i}_{R, 1} e^{j \theta_{m}} \bar{i}_{S, 1}^{*}\right\}
$$

where ${ }^{*}$ is used to indicate the conjugate operator $\left(j^{*}=-j\right)$, and $p$ is the number of pole pairs. Equations (5)-(11), together with the equation of motion, can be used to evaluate the electromechanical behavior of the machine.

\section{Model of a Twelve-Phase Induction Machine}

Replacing (7)-(10) in (5)-(6) leads to the following equations, which are valid for a twelve-phase induction machine:

$$
\begin{gathered}
\bar{v}_{S, 1}=R_{S} \bar{i}_{S, 1}+L_{S, 1} \frac{d \bar{i}_{S, 1}}{d t}+M_{1} \frac{d\left(\bar{i}_{R, 1} e^{j \theta_{m}}\right)}{d t} \\
0=R_{R, 1} \bar{i}_{R, 1}+L_{R, 1} \frac{d \bar{i}_{R, 1}}{d t}+M_{1} \frac{d\left(\bar{i}_{S, 1} e^{-j \theta_{m}}\right)}{d t} \\
\bar{v}_{S, \rho}=R_{S} \bar{i}_{S, \rho}+l_{S} \frac{d \bar{i}_{S, \rho}}{d t}, \quad \rho=3,5,7,9,11 .
\end{gathered}
$$

Equations (12)-(14) can be used to analyze the behavior of the different layouts of a twelve-phase machine $(m=12)$. The stator windings can be connected to each other in different ways, depending on the number of insulated neutral points. For example, the possible connections of four three-phase windings are shown in Fig. 2. On the one hand, the singlestar layout presents more degrees of freedom in the current control and is of interest for the implementation of control systems based on high-order field harmonics or diagnostic algorithms that employ the same harmonics to obtain information on the parameters and the behavior of the electrical

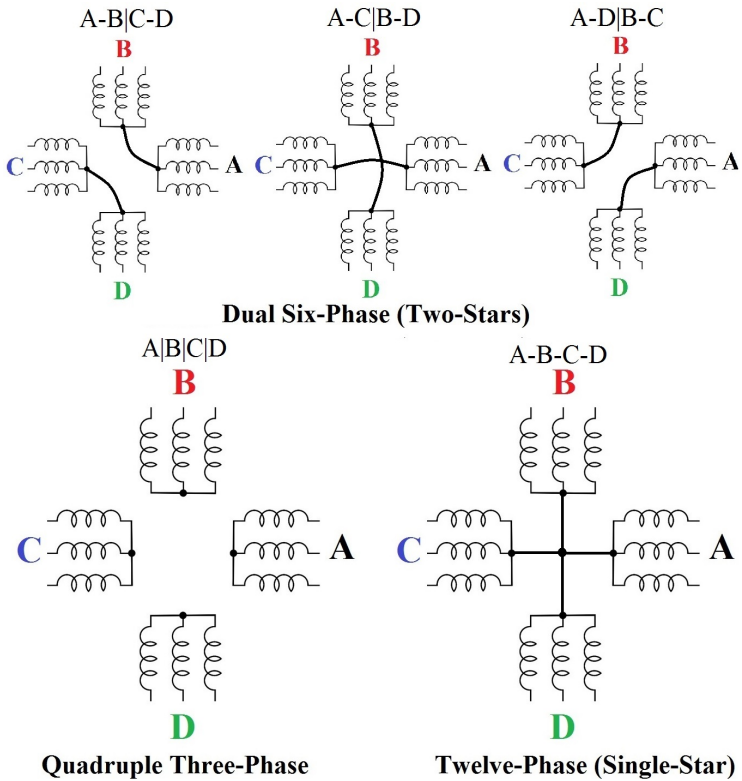

Fig. 2. Most interesting star connection layouts of a twelve-phase distributedwinding machine.

machine. This layout is also the one that allows the best postfault performance of the drive. On the other hand, the multi three-phase solution features galvanic insulation of the subwindings. This insulation allows supplying each sub-winding with an independent inverter connected to a separated DC link, resulting in a modular converter architecture that is suitable for the implementation of power-sharing control schemes and the employment of conventional three-phase inverter technologies. Intermediate solutions (as the double six-phase layouts in Fig. 2) represent possible alternatives to mediate between the two aforementioned opposite architectures.

\section{Constraints due to the Star Connections}

In a machine with $m$ phases and $N_{s t}$ insulated neutral points (or stars), the degrees of freedom in the control of the phase currents are $m-N_{s t}$. The phases of the $h t h$ star-connected winding are those whose indexes belong to the set $W_{h}$. Owing to the star connections, the sum of the currents flowing toward the same neutral point is zero:

$$
\sum_{k \in W_{h}} i_{k}=0, \quad h=1,2, \ldots, N_{s t} .
$$

By introducing the inverse transformation (3) or (4), it is possible to express the constraints described by (15) in terms of current SVs. It turns out that only some current SVs are constrained by (15), whereas the fundamental current $\mathrm{SV} \bar{i}_{1}$ is not affected, because it vanishes from the equation. As a result, it is possible to rewrite (15) in terms of SV components by using the following matrix notation:

$$
C \mathbf{i}_{a u x}=0
$$

where $C$ is a full-rank $N_{s t}$-by- $(m-2)$ matrix, and $\mathbf{i}_{\text {aux }}$ is the column vector of the $\alpha-\beta$ components of the current 
SVs different from $\bar{i}_{1}$. The expression of $\mathbf{i}_{\text {aux }}$, respectively for systems with odd and even numbers of phases, is as follows:

$$
\begin{aligned}
& \mathbf{i}_{\text {aux }}=\left[\begin{array}{lllllll}
i_{3 \alpha} & i_{3 \beta} & i_{5 \alpha} & i_{5 \beta} & \ldots & \ldots & i_{m \alpha(\beta)}
\end{array}\right]^{\top} \\
& \mathbf{i}_{a u x}=\left[\begin{array}{lllllll}
i_{3 \alpha} & i_{3 \beta} & i_{5 \alpha} & i_{5 \beta} & \ldots & \ldots & i_{(m-1) \alpha} \\
i_{(m-1) \beta}
\end{array}\right]^{\top}
\end{aligned}
$$

where the operator " $T$ " identifies the transpose operator. The SVs $\bar{i}_{\rho}$ with $\rho>1$ are called auxiliary vectors to distinguish them from the fundamental one $\bar{i}_{1}$, which can be rewritten as a column vector as follows:

$$
\mathbf{i}_{1}=\left[\begin{array}{ll}
i_{1 \alpha} & i_{1 \beta}
\end{array}\right]^{\top} .
$$

Equation (16) is used in Section III, together with the constraints resulting from an open-phase fault, in order to define an optimized post-fault algorithm.

\section{E. Model of a Multiphase Stator Winding in Case of Open- Phase Fault}

The zeroing of a stator current may be caused by a fault in the converter or the machine, as shown in Fig. 3 for a standard three-phase drive. The breakdown of the switching devices or the activation of the active and passive protections of the drive (e.g., the desaturation protection, that turns the switches off in order to avoid dangerous over-currents) can easily cause the uncontrolled phase currents to be zero. Also, this happens in case of actual open-phase faults due to the disconnection of the phases from the terminal box, the neutral point or the terminals of the inverter. This might result from assembly mistakes or the deterioration of the connections.

In the post-fault condition, the machine operates under the constraints that the currents of the faulty phases $k_{1}, k_{2}, \ldots k_{N_{f}}$ are zero:

$$
i_{k_{f}}=0 \quad f=1,2, \ldots N_{f} .
$$

In terms of current SVs, (20) can be rewritten using the inverse Clarke's transformation (3) or (4) and expressed in matrix form as follows:

$$
B \mathbf{i}_{1}+A \mathbf{i}_{\text {aux }}=0
$$

where $B$ is an $N_{f}$-by- 2 matrix, and $A$ is an $N_{f}$-by- $(m-2)$ matrix, respectively defined as follows:

$$
B=\left[\begin{array}{cccc}
c_{1, k_{1}} & \ldots & \ldots & c_{1, k_{N_{f}}} \\
s_{1, k_{1}} & \ldots & \ldots & s_{1, k_{N_{f}}}
\end{array}\right]^{\top}
$$

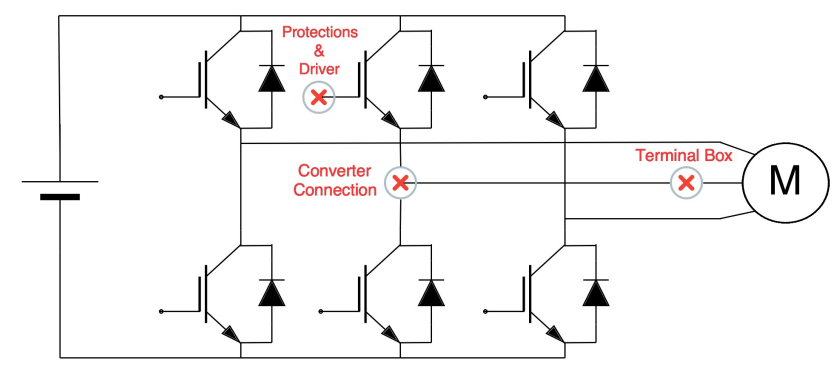

Fig. 3. Open-phase fault typologies.

$$
A=\left[\begin{array}{cccc}
c_{3, k_{1}} & \ldots & \ldots & c_{3, k_{N_{f}}} \\
s_{3, k_{1}} & \ldots & \ldots & s_{3, k_{N_{f}}} \\
c_{5, k_{1}} & \ldots & \ldots & c_{5, k_{N_{f}}} \\
s_{5, k_{1}} & \ldots & \ldots & s_{5, k_{N_{f}}} \\
\ldots & \ldots & \ldots & \ldots \\
\ldots & \ldots & \ldots & \ldots \\
c_{(m-1)_{\text {odd }}, k_{1}} & \ldots & \ldots & c_{(m-1)_{o d d}, k_{N_{f}}} \\
s_{(m-1)_{o d d}, k_{1}} & \ldots & \ldots & s_{(m-1)_{o d d}, k_{N_{f}}} \\
c_{(m)_{\text {odd }}, k_{1}} & \ldots & \ldots & c_{(m)_{o d d}, k_{N_{f}}}
\end{array}\right]^{\top}
$$

where $c_{\rho, k_{f}}=\cos \left(\rho \varphi_{k_{f}}\right)$ and $s_{\rho, k_{f}}=\sin \left(\rho \varphi_{k_{f}}\right)$, and $\varphi_{k_{f}}$ is the electrical angle of the faulty phase with index $k_{f}$, as defined in (2).

In the next section, the post-fault constraint (21) is combined with the one resulting from the presence of insulated neutral points (16) in order to find the analytical solution for the optimized post-fault algorithm.

\section{Post-Fault Control Algorithm with Minimum COPPER JOUle LOSSES}

Managing an open-phase fault is essential to ensure the operation of the drive (e.g., to avoid instability phenomena or unexpected extra losses) and to optimize its post-fault performance. Furthermore, such a control scheme could be used to keep the current of one or more phases at zero in case of necessity, although a fault has not occurred yet. The advantages of zeroing the currents in particular phases are twofold: reduce the related copper losses and minimize the stress of the emergency AC breakers for the disconnection of these phases. For example, if a fault or a localized temperature increase is detected, the current of the phase placed next to that area can be controlled to zero in order to avoid the accelerated aging of the insulation. Furthermore, in case of a redundant converter architecture (as in a multi three-phase configuration), it would be possible to control to zero the power of one subconverter, so that its DC link could be opened with a limited effort and without incurring in the phenomenon of electric arc. Then, the converter could be replaced, or its maintenance could be carried out without unscheduled service interruption. Because of the importance of the open-phase fault-tolerant algorithm in highly reliable drives, this section shows a general approach to define an optimized post-fault algorithm for a multiphase machine analytically. The fundamental contributions to the electromagnetic torque and flux depend on $\bar{i}_{1}$, which can be assumed as a known quantity calculated by the control system depending on the operating conditions. Therefore, the post-fault control problem consists in finding a solution for $\mathbf{i}_{\text {aux }}$ that satisfies (16) and (21). The system of equations obtained combining (16) and (21) has $N_{s t}+N_{f}$ rows and $m-2$ unknown variables, so it is usually under-determined being $N_{s t}+N_{f}<m-2$. Among the possible solutions of (16) and (21), the one that minimizes the total stator copper losses can be found analytically.

The stator copper losses are as follows:

$$
P_{J}=\sum_{k=1}^{m} R_{s} i_{k}^{2}=\frac{m}{2} R_{s} \mathbf{i}_{1}^{\top} \mathbf{i}_{1}+\frac{m}{2} R_{s} \mathbf{i}_{\text {aux }}^{\top} \mathbf{i}_{\text {aux }} .
$$


The solution to the problem of minimizing (24) with the constraints (16) and (21) can be written in closed form, as explained hereafter.

Let $D$ be the $\left(N_{s t}+N_{f}\right)$-by- $(m-2)$ matrix obtained by appending the rows of $C$ to the rows of $A$,

$$
D=\left[\begin{array}{l}
A \\
C
\end{array}\right]
$$

and let $E$ be the $\left(N_{s t}+N_{f}\right)$-by-2 matrix obtained by appending $N_{s t}$ null rows to the rows of $B$.

$$
E=\left[\begin{array}{c}
B \\
0_{\left(N_{s t}, 2\right)}
\end{array}\right] \text {. }
$$

The rank of $D$ is $\left(N_{s t}+N_{f}\right)$. The set of equations (16) and (21) can be written in compact form as follows:

$$
D \mathbf{i}_{a u x}+E \mathbf{i}_{1}=0 .
$$

The solution to the optimization problem is

$$
\mathbf{i}_{\text {aux }}=F \mathbf{i}_{1}
$$

where $F$ is a $(m-2)$-by-2 matrix defined as follows:

$$
F=-D^{+} E \text {. }
$$

The operator "+" applied to $D$ in (29) represents the MoorePenrose inverse of $D$, more precisely its right pseudo-inverse matrix defined as follows:

$$
D^{+}=D^{\top}\left(D D^{\top}\right)^{-1}
$$

The resulting matrix $F$ represents a unique set of constants, which relates the auxiliary SVs $\mathbf{i}_{a u x}$ to $\mathbf{i}_{1}$ for each fault configuration and layout of the neutral points and minimizes the stator copper losses. The values of $F$ are not affected by the operating condition of the machine and need to be calculated only once, when the fault is detected. Also, these values can be calculated offline and stored on a conventional control platform such as a DSP. As an example, the aspect of matrices $A, B$, $C$ and $F$ is analyzed in the Appendix and presented in Table III for a particular fault scenario.

In a machine without star connections, i.e., a multi singlephase or an open-end winding layout, the matrix $F$ in (28)(29) can be simplified as follows:

$$
F=-A^{\top}\left[A A^{\top}\right]^{-1} B
$$

When the machine has an odd number of symmetrically distributed phases and a single star, $C$ is a single row matrix. The entry in the $(m-2) t h$ column is 1 , while the other entries are zero. In other terms, it is enough to neglect the one-dimension current space vector component related to the zero-sequence current in the $A$ matrix (i.e., the $m t h$ column of $A$ ). In this case, in agreement with the relationship found in [11], (31) can still be used. In conclusion, (28) can be employed to directly find the optimal solution for symmetrical or asymmetrical multiphase windings with different layouts of the star connections and in any open-phase post-fault operation.

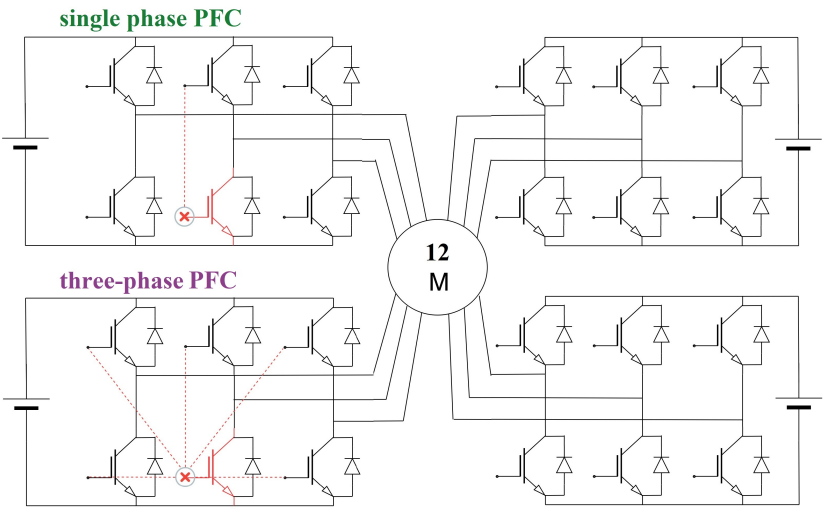

Fig. 4. Open-phase fault management in a quadruple three-phase converter: "single-phase PFC" logic (left top) and "three-phase PFC" logic (left bottom).

\section{Simplified Post-Fault Control Algorithm For MULTI $n$-PHASE DRIVES}

This section briefly summarizes a simplified control algorithm suitable for multi $n$-phase windings. The algorithm is based on an analytical approach and is suitable for drives with a high number of phases and star connections (e.g., a quadruple three-phase one) where the application admits a high reduction in the machine performance in the post-fault operation.

The problem of Post-Fault Control (PFC) is linked to the concept of power-sharing. According to this technique, the overall power of a multi $n$-phase drive can be unequally split among a set of independent $n$-phase converters [24], [29][31]. A particular case of power-sharing is when the output power (or current) of a converter is zero. This case perfectly matches the post-fault control of the machine.

Different PFC strategies can be defined, as shown in Fig. 4 for a quadruple three-phase electric drive. After the failure of one device, two strategies seem more reasonable. The first one consists in disabling the switches of the faulty phase ("single-phase PFC"); the second one consists in disabling all the switches of the inverter involved in the fault ("three-phase PFC"). When commercial three-phase inverters are used, the latter approach is simpler. The three-phase PFC strategy can be also preferred because it avoids the low frequency distortion of the power flows at the DC links of the converter in case the converter is built of independent modules connected to insulated DC links.

The next section shows the proposed control architectures of a twelve-phase induction motor drive for various winding layouts and compares them in terms of control design and performance.

\section{Control Architecture}

A standard three-phase machine is controlled through the fundamental SV $\bar{i}_{S, 1}$. To control this current vector, two Proportional-Integral (PI) regulators are usually employed. In general, the minimum number of PI regulators required to control the current SVs of an $m$-phase machine with $N_{s t}$ stars is equal to the number of independently controllable currents, i.e., $m-N_{s t}$. 


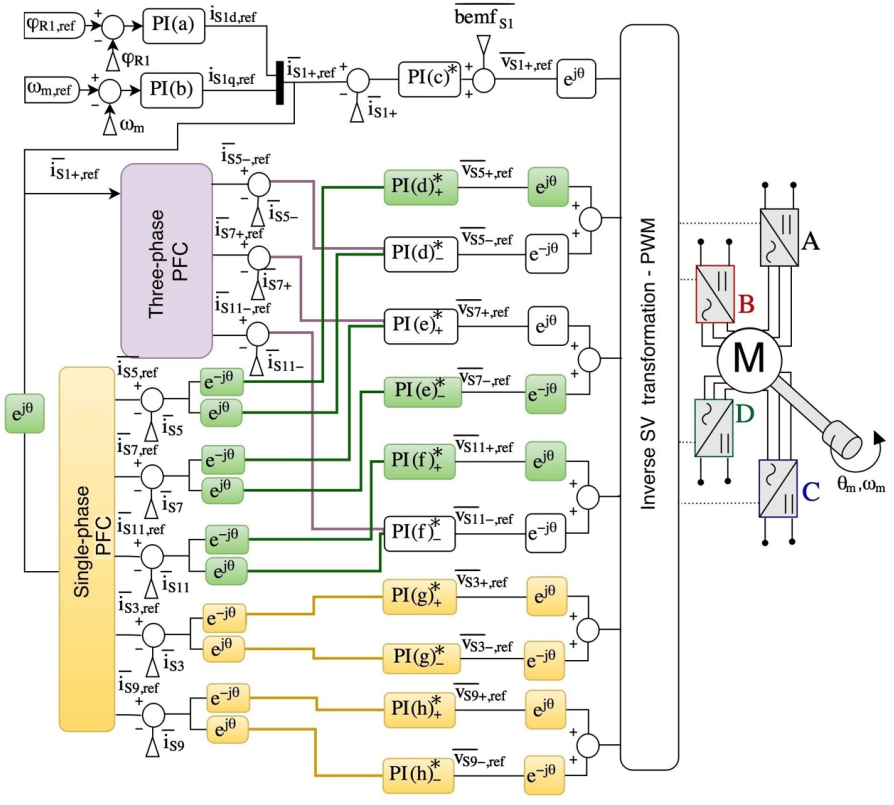

Fig. 5. Control algorithm of a twelve-phase machine in different conditions: healthy or "three-phase PFC" with four insulated neutral points (white), faulty with four independent stars and "single-phase PFC" (white and green), healthy with double six-phase or single star (white and yellow), faulty with double six-phase or single star and "single-phase PFC" (white, green and yellow).

For example, Fig. 5 shows the control scheme of a quadruple three-phase machine $\left(m=12\right.$ and $\left.N_{s t}=4\right)$. The signals at the input of the PI controllers are complex quantities, so each block highlighted with a "*” in Fig. 5 must be intended as a couple of PI regulators, respectively for the $d$ and $q$ components of the signals. The eight PI current controllers colored in white are the ones required to control the healthy machine $\left(m-N_{s t}=8\right)$. Two current PI controllers are required to track the reference set points of the fundamental space, whereas the other six PI controllers are used to keep at zero the currents in the harmonic sub-spaces.

Conversely, if all the sub-windings were connected together to the same neutral point ( $m=12$ and $N_{s t}=1$, i.e., $\left.m-N_{s t}=11\right)$ the number of PI regulators needed to control the machine would increase to $m-1$, i.e., 11 .

However, a post-fault algorithm requires, in general, more PI regulators than the normal control system. In fact, the trajectories of some current SVs are often elliptic (even degenerate) rather than circular. For each SV with an elliptic trajectory, it is necessary to use a pair of PI regulators, implemented in counter-rotating reference frames, in order to ensure a theoretical zero-error at steady state. Hence, the PI regulators for the control of these SVs doubles. Although for each faulty phase the number of the degrees of freedom decreases by one, it results that the number of the PI regulators for the control scheme of the faulty machine needs to be increased to obtain an effective tracking of the current references.

It is possible to analyze how the control architecture changes depending on the layout of the winding, the type of fault and the adopted post-fault strategy. Under the assumption of an open-phase fault, the control scheme of a quadruple threephase machine does not require any additional PI regulator if the "three-phase PFC" is implemented (purple signals in Fig. 5). Conversely, if the optimized "single-phase PFC" is used, the control system requires three additional pairs of PI regulators (green signals at the input of regulators $d$, e and $f$ ). Also, if the winding layout is a single-star type or a double six-phase type, the number of PI regulators required to implement the "single-phase PFC" increases further. The additional regulators are necessary to control the zero-sequence currents flowing from and to different three-phase windings. In Fig. 5, the additional PIs needed in case of "single-phase PFC" are highlighted in yellow. Hence, the total number of PI regulators for the current control raises to 11 pairs.

\section{Analytical Results}

In this section, the post-fault strategies and star connection layouts are analyzed for the quadruple three-phase induction machine in a single-phase post-fault operation.

The comparison is carried out in terms of stator copper losses and maximum phase currents by varying the magnitude of the fundamental current SV $\left|\bar{i}_{S, 1}\right|$. Table I presents the main machine parameters.

Fig. 6 and 7 show the stator copper losses and the maximum phase currents respectively, as a function of the magnitude of the main current SV $\left|\bar{i}_{S, 1}\right|$ and for the analyzed post-fault controls and star connection layouts. It is possible to notice from Fig. 6 (green line) that for a quadruple three-phase layout $A|B| C \mid D$ the rated copper losses are reached at about 14.81 $\mathrm{A}$ and $13.86 \mathrm{~A}$ in case of a single-phase open-fault with a "single-phase PFC" and a "three-phase PFC" respectively, compared with 16 A $\left|\bar{i}_{S, 1}\right|$ in healthy conditions. Conversely, assuming a "single-star PFC" the rated losses are reached at about $15.18 \mathrm{~A}$ with a single star layout, and at about $15.08 \mathrm{~A}$ for all the double six-phase layouts.

Fewer differences between the single-phase and the threephase PFCs have been found in terms of maximum phase currents. In fact, as illustrated in Fig. 7, the limitation on the peak current during post-fault operation leads to a reduction of the maximum magnitude of $\bar{i}_{S, 1}$ from $23 \mathrm{~A}$, in healthy conditions, to about 17.5 A during the post-fault operation. A worse result has been found for the double six-phase layouts. The $A-B \mid C-D$ layout admits a maximum $\left|\bar{i}_{S, 1}\right|$ value of $15.54 \mathrm{~A}$, which increases to $16.16 \mathrm{~A}$ and 17.21 $\mathrm{A}$ for the

TABLE I

MAIN MAChine PARAMETERs (50 Hz)

\begin{tabular}{lll}
\hline \hline \multicolumn{1}{c}{ Symbol } & \multicolumn{1}{c}{ Quantity } & \multicolumn{1}{c}{ Value } \\
\hline$P_{\text {rated }}$ & rated power & $10 \mathrm{~kW}$ \\
$T_{\text {rated }}$ & rated torque & $16 \mathrm{Nm}$ \\
$\left|\bar{i}_{S, 1, \text { rated }}\right|$ & main current vector magnitude & $16 \mathrm{~A}_{p k}$ \\
$i_{S, 1, d, \text { rated }}$ & rated d-axis current & $10 A_{p k}$ \\
$I_{\text {max }}$ & maximum phase current & $23 A_{p k}$ \\
$\left|\bar{v}_{S, 1, \text { rated }}\right|$ & rated phase voltage & $186 V_{p k}$ \\
$R_{S}$ & stator resistance & $0.188 \Omega$ \\
$R_{R, 1}$ & rotor resistance for the subspace 1 & $0.156 \Omega$ \\
$L_{S, 1}, L_{R, 1}$ & stator and rotor inductances & $12.8 \mathrm{mH}$ \\
$M_{1}$ & mutual inductance & $12.0 \mathrm{mH}$ \\
$p$ & number of pole pairs & 2 \\
\hline \hline
\end{tabular}




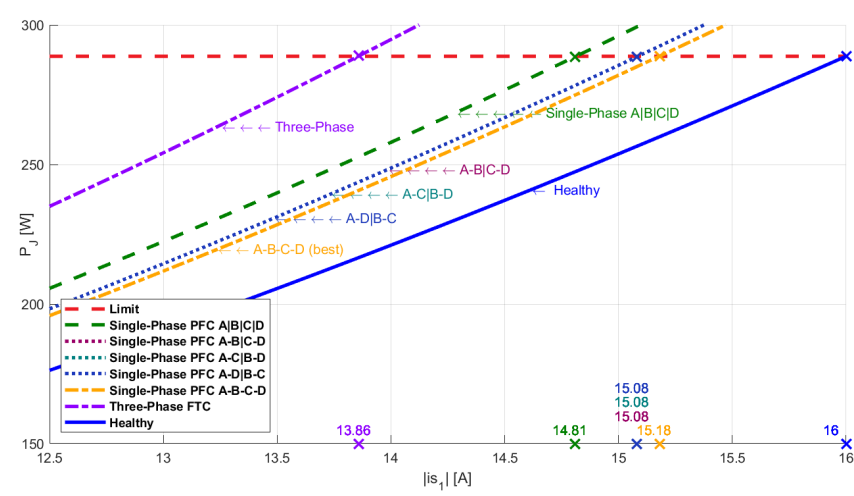

Fig. 6. Comparison of analytical stator copper losses with the healthy machine (blue) and the faulty machine (phase A1 open). "Three-phase PFC" (purple) and "single-phase PFC": quadruple three-phase layout (green), double sixphase layouts (spotted) and twelve-phase single star layout (yellow). The rated copper losses are highlighted in red.

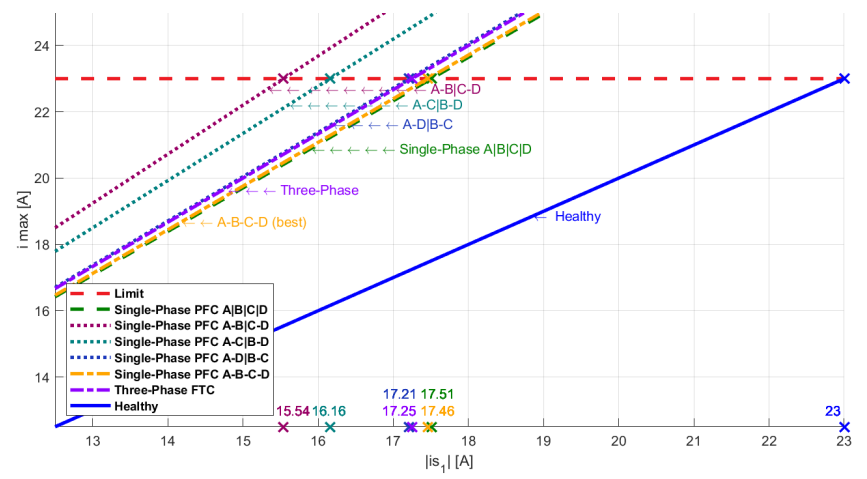

Fig. 7. Comparison of analytical maximum phase current with the healthy machine (blue) and the faulty machine (phase A1 open). "Three-phase PFC" (purple) and "single-phase PFC": quadruple three-phase layout (green), double six-phase layouts (spotted) and twelve-phase single star layout (yellow). The maximum phase current is highlighted in red.

layouts $A-C \mid B-D$ and $A-D \mid B-C$, respectively. It is worth to notice that the only result that changes with the position of the faulty phase is the peak current in the double six-phase layout $A-D \mid B-C$, that in half of the cases results in the same constraint obtained for the layout $A-B \mid C-D$ (i.e., it admits a maximum $\left|\bar{i}_{S, 1}\right|$ value of $15.54 \mathrm{~A}$ ). Considering the worse condition in terms peak current for all the possible open-phase faults, it turns out that $A-C \mid B-D$ is the dual sixphase layout admits the highest set point for the fundamental current vector $\left|\bar{i}_{S, 1}\right|$.

In the proposed control technique, the control system ensures that the magnitude of the current space vector in the fundamental subspace is always kept below a threshold value that makes the phase currents satisfy the current constraint. This threshold value depends on the fault scenario. The auxiliary current space vectors are indirectly limited, being calculated from the fundamental one by (28).

In case of healthy machine, the current limitation affects the fundamental current vector (which is the only one controlled to a value that differs from zero) and is equal to the inverters' current limit.

In case of a fault, the limitation of the fundamental current space vector must consider the different peak values that are

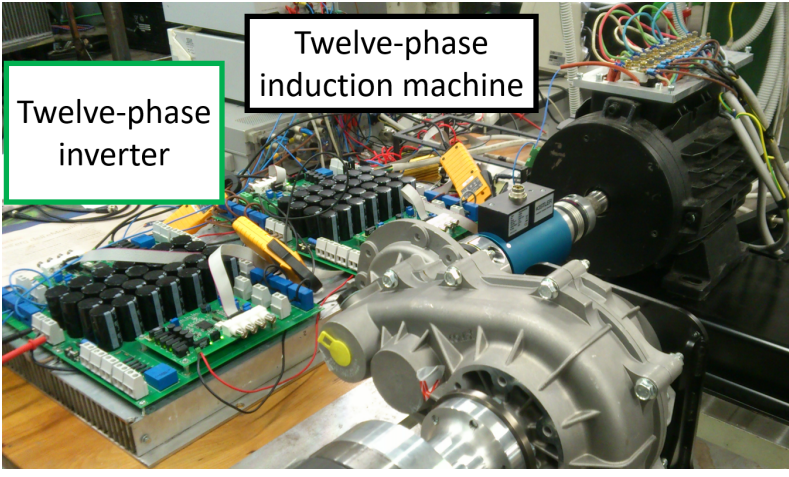

Fig. 8. Test bench: twelve-phase inverter (left) and machine prototype (right).

reached from the remaining healthy phases. For example, for the considered machine with a rated inverter current of 23 A, the limitation of the fundamental current vector in case of a single open-phase fault corresponds to the values that are reported in Fig. 7 above the horizontal axis.

The comparisons of the stator copper losses and the peak currents show that the proposed post-fault control allows the continuous operation of the machine with an acceptable reduction of power. In contrast, the available overload capability is limited by a tighter constraint related to the maximum peak of the phase currents.

\section{EXPERIMENTAL RESULTS}

In order to experimentally assess the proposed control strategy for five different winding layouts of multiphase drive, a prototype of a quadruple three-phase induction machine has been employed. The test bench, the quadruple three-phase induction machine and the multiphase inverter, commanded by a DSP TMS320F28335 controller, are shown in Fig. 8.

Fig. 9 illustrates the waveforms of the stator currents and the trajectories of the current SVs in the $\alpha-\beta$ planes when the machine operates in an open-phase fault condition (the first phase of the inverter $\mathrm{A}$ is open). The experimental results are presented for the most significant post-fault control strategies (the optimum "single-phase PFC", and the simpler "three-phase PFC") and star-connection layouts (quadruple three-phase, double six-phase and single-star twelve-phase configurations). The machine is operating at $700 \mathrm{rpm}$. The torque is $7.5 \mathrm{Nm}$ and $i_{S, 1, d}$ is $10 \mathrm{~A}$.

The current waveforms and trajectories of the current SVs are as expected from the analytical study, except for the current ripple. The distortion of the phase currents is mainly attributed to the effects of the high-order field harmonics and the machine and converter nonlinearities. The peak values of the phase currents in the results of Fig. 9 do not show significant variations when different star-connection layouts or an improved "single-phase PFC" algorithm are used. This agrees with the results presented in Fig. 7. Table II summarizes the experimental results in terms of stator copper losses and number of additional PI regulators required for effective control of the current SVs (in case of healthy and faulty machine). The results are in agreement with the expected analytical solutions with a maximum mismatch on the stator 
Healthy (quadruple three-phase)

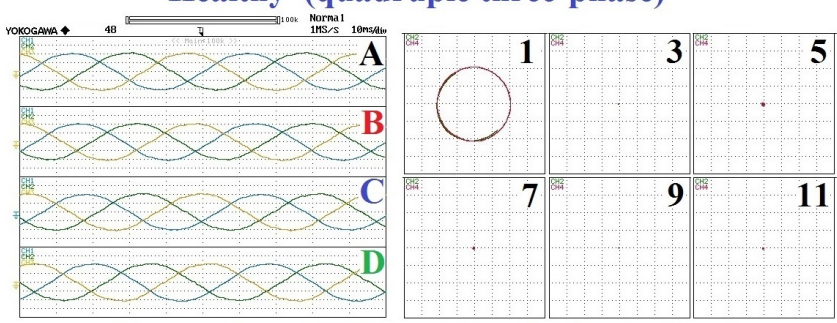

Three-phase PFC (quadruple three-phase)

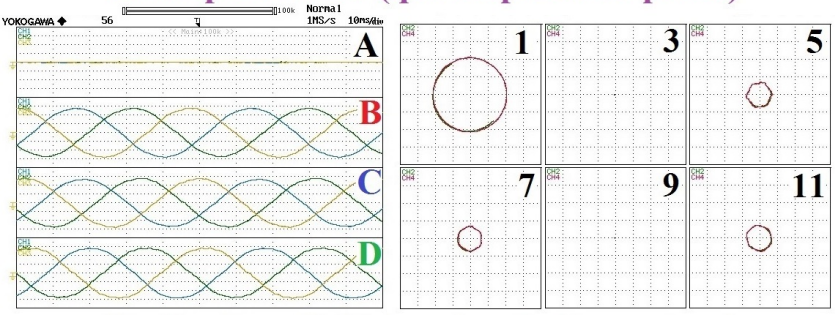

Single-phase PFC (quadruple three-phase)

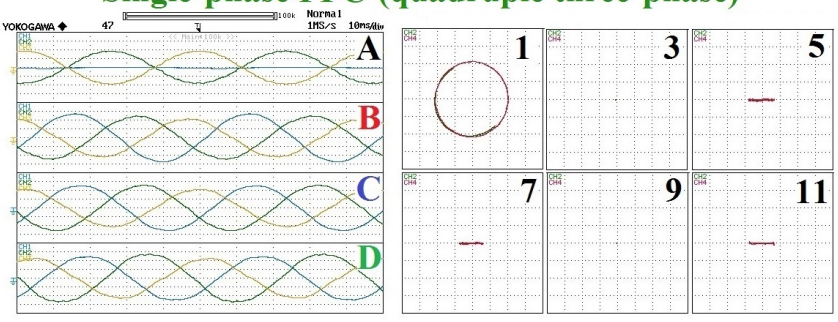

Single-phase PFC (double six-phase A-C $\mid \mathrm{B}-\mathrm{D}$ )
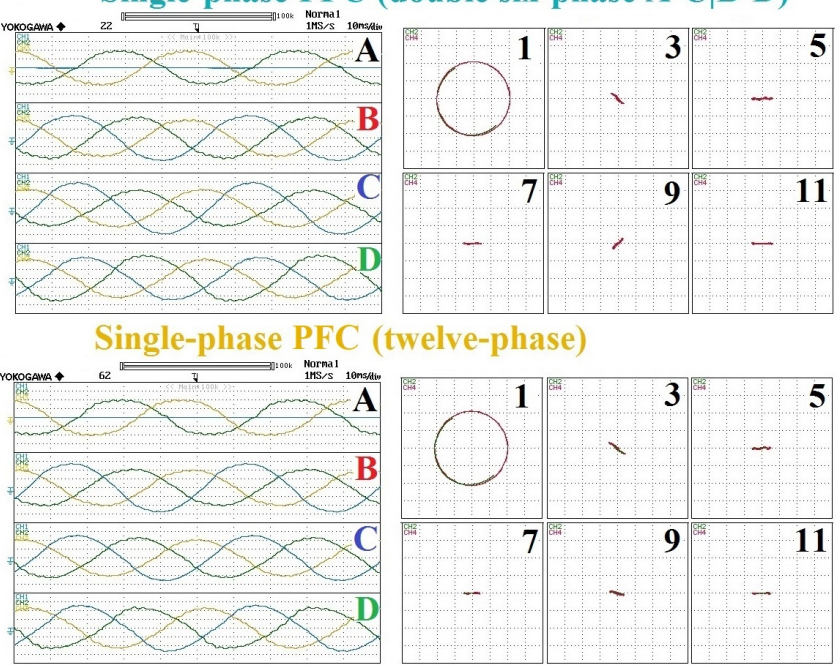

Fig. 9. Phase currents (left) and current SV trajectories (right, with in red the measured values and in green the reference values) when the machine is operating in healthy operation (first row), and in the same post fault condition (phase A1 open fault) with the most significant post-fault controls and star connection layouts. The scale is $5 \mathrm{~A} / \mathrm{div}$ in all the sub figures.

copper losses of $4.6 \%$. In terms of stator copper losses, the comparison shows significant advantages in using a single star layout and a "single-phase PFC" over a quadruple three-phase layout and a "three-phase PFC". However, this improvement is obtained with a more complicated control architecture. The other possible star layouts and post-fault algorithms present intermediate solutions, and the selection of the layout has to be evaluated according to the considered application and its fault-tolerant requirements.
TABLE II

COMPARISON OF THE POST-FAULT CONTROLS (THREE-PHASE, 3-PH, AND SINGLE-PHASE, 1-PH, POST-FAULT CONTROLS) AND STAR CONNECTION LAYOUTS FOR A SINGLE-PHASE OPEN FAULT

\begin{tabular}{lll}
\hline \hline & $\begin{array}{l}\text { Copper losses W } \\
\text { (experimental) } \\
0.188 \Omega \text { resistance }\end{array}$ & $\begin{array}{l}\text { Computational effort in } \\
\text { N. of PIs (N. of additional } \\
\text { PIs vs. the healthy quadruple } \\
\text { three-phase layout) }\end{array}$ \\
\hline Healthy (quadruple 3-ph) & $140.3(+0 \%)$ & 8 \\
Healthy (double 6-ph) & $140.9(+0 \%)$ & $12(+4)$ \\
Healthy (12-ph) & $140.4(+0 \%)$ & $12(+4)$ \\
3-ph PFC (quadruple 3-ph) & $181.5(+29.3 \%)$ & $8(+0)$ \\
3-ph PFC (double 6-ph) & $181.1(+28.5 \%)$ & $12(+4)$ \\
3-ph PFC (12-ph) & $178.5(+27.1 \%)$ & $12(+4)$ \\
1-ph PFC (quadruple 3-ph) & $160.7(+14.5 \%)$ & $14(+6)$ \\
1-ph PFC (double 6-ph) & $156.4(+11.0 \%)$ & $16(+8)$ \\
1-ph PFC (12-ph) & $153.8(+9.5 \%)$ & $16(+8)$ \\
\hline \hline
\end{tabular}

In case of a higher number of faulty phases, the advantage of using a more complicated control strategy with a reduced number of neutral points seems even more significant in terms of copper losses. However, the probability of simultaneous open-phase faults in different converters is much lower than a single-phase or a single-converter failure. Therefore, the presented analysis and the developed control strategy, together with the experimental validation summarized in Table II, can be considered as a useful guideline for the choice of the faulttolerant design/control architecture of a multiphase drive.

\section{CONCLUSION}

This paper extends the post-fault control algorithms developed for multiphase drives under open-phase fault operation to multiphase machines with any layout of the star connections. The presented post-fault strategy, based on the minimization of the stator copper losses, allows defining the optimum reference currents for symmetrical or asymmetrical multiphase windings with different star connection layouts and in any open-phase post-fault operation, under the assumption of neglecting the effects of the high-order field harmonics. In fact, (28) can be directly implemented on a control platform in each fault scenario, and the optimum post-fault algorithm is calculated without the need for numerical iterations. The proposed method takes into account the constraint introduced by the different star-connection layouts of the $n$-phase subwindings, highlighting advantages and drawbacks in terms of stator copper losses, peak currents and computational efforts (number of additional PI regulators) resulting from the winding architecture and post-fault strategy.

The theoretical considerations have been verified by experimental tests using a prototype of a quadruple three-phase induction machine. This work aims to give a contribution to the state of the art in the design and control of multiphase machines for safety-critical applications.

\section{APPENDIX}

The matrices $A, B, C$ and $F$ for a particular open-phase fault (phase A2) are reported in Table III. In particular, the matrices $A, B$ and $C$ are used in (25)-(29) to determine the post-fault matrix $F$. All these matrices are constant and are 
only related to the winding layout, the fault configuration and post fault strategy. Thus, they are not affected by the actual operation of the drive.

\section{REFERENCES}

[1] J. W. Bennett, B. C. Mecrow, D. J. Atkinson, and G. J. Atkinson, "Safety-critical design of electromechanical actuation systems in commercial aircraft," IET Electric Power Applications, vol. 5, no. 1, pp. 37-47, 2011.

[2] V. Madonna, P. Giangrande, and M. Galea, "Electrical power generation in aircraft: Review, challenges, and opportunities," IEEE Transactions on Transportation Electrification, vol. 4, no. 3, pp. 646-659, 2018.

[3] J. W. Bennett, G. J. Atkinson, B. C. Mecrow, and D. J. Atkinson, "Fault-tolerant design considerations and control strategies for aerospace drives," IEEE Transactions on Industrial Electronics, vol. 59, no. 5, pp. 2049-2058, 2012.

[4] P. Zheng, Y. Sui, J. Zhao, C. Tong, T. A. Lipo, and A. Wang, "Investigation of a novel five-phase modular permanent-magnet in-wheel motor," IEEE Transactions on Magnetics, vol. 47, no. 10, pp. 40844087, 2011.

[5] G. Feng, C. Lai, W. Li, J. Tjong, and N. C. Kar, "Open-phase fault modeling and optimized fault-tolerant control of dual three-phase permanent magnet synchronous machines," IEEE Transactions on Power Electronics, vol. 34, no. 11, pp. 11 116-11 127, 2019.

[6] D. T. Vu, N. K. Nguyen, E. Semail, and T. J. dos Santos Moraes, "Control strategies for non-sinusoidal multiphase pmsm drives in faulty modes under constraints on copper losses and peak phase voltage," IET Electric Power Applications, vol. 13, no. 11, pp. 1743-1752, 2019.

[7] F. Yu, M. Cheng, and K. T. Chau, "Controllability and performance of a nine-phase fspm motor under severe five open-phase fault conditions," IEEE Transactions on Energy Conversion, vol. 31, no. 1, pp. 323-332, 2016.

[8] X. Wang, Z. Wang, Z. Xu, J. He, and W. Zhao, "Diagnosis and tolerance of common electrical faults in t-type three-level inverters fed dual threephase pmsm drives," IEEE Transactions on Power Electronics, vol. 35, no. 2, pp. 1753-1769, 2020.

[9] T. S. de Souza, R. R. Bastos, and B. J. Cardoso Filho, "Synchronousframe modeling and $\mathrm{dq}$ current control of an unbalanced nine-phase induction motor due to open phases," IEEE Transactions on Industry Applications, vol. 56, no. 2, pp. 2097-2106, 2020.

[10] X. Wang, Z. Wang, M. Gu, D. Xiao, J. He, and A. Emadi, "Diagnosisfree self-healing scheme for open-circuit faults in dual three-phase pmsm drives," IEEE Transactions on Power Electronics, pp. 1-1, 2020.

[11] A. Tani, M. Mengoni, L. Zarri, G. Serra, and D. Casadei, "Control of multiphase induction motors with an odd number of phases under opencircuit phase faults," IEEE Transactions on Power Electronics, vol. 27, no. 2, pp. 565-577, 2012

[12] H. S. Che, M. J. Duran, E. Levi, M. Jones, W. P. Hew, and N. A. Rahim, "Postfault operation of an asymmetrical six-phase induction machine with single and two isolated neutral points," IEEE Transactions on Power Electronics, vol. 29, no. 10, pp. 5406-5416, 2014.

[13] W. N. W. A. Munim, M. J. Duran, H. S. Che, M. Bermudez, I. GonzalezPrieto, and N. A. Rahim, "A unified analysis of the fault tolerance capability in six-phase induction motor drives," IEEE Transactions on Power Electronics, vol. 32, no. 10, pp. 7824-7836, 2017.

[14] F. Baneira, J. D. Gandoy, A. G. Yepes, O. Lopez, and D. P. Estevez, "Control strategy for multiphase drives with minimum losses in the full torque operation range under single open phase fault," IEEE Transactions on Power Electronics, vol. 32, no. 8, pp. 6275-6285, 2017.

[15] A. G. Yepes, J. Doval-Gandoy, F. Baneira, and H. A. Toliyat, "Comparison of stator winding connections in multiphase drives under healthy operation and with one open converter leg," IET Electric Power Applications, vol. 14, no. 4, pp. 584-596, 2020.

[16] A. S. Abdel-Khalik, S. Ahmed, and A. M. Massoud, "Steady-state equivalent circuit of five-phase induction machines with different stator connections under open-line conditions," IEEE Transactions on Industrial Electronics, vol. 63, no. 8, pp. 4651-4662, 2016.

[17] A. S. Abdel-Khalik, A. S. Morsy, S. Ahmed, and A. M. Massoud, "Effect of stator winding connection on performance of five-phase induction machines," IEEE Transactions on Industrial Electronics, vol. 61, no. 1, pp. 3-19, 2014.

[18] I. Gonzalez-Prieto, M. J. Duran, H. S. Che, E. Levi, M. Bermudez, and F. Barrero, "Fault-tolerant operation of six-phase energy conversion systems with parallel machine-side converters," IEEE Transactions on Power Electronics, vol. 31, no. 4, pp. 3068-3079, 2016.
[19] A. S. Abdel-Khalik, M. S. Hamad, A. M. Massoud, and S. Ahmed, "Postfault operation of a nine-phase six-terminal induction machine under single open-line fault," IEEE Transactions on Industrial Electronics, vol. 65, no. 2, pp. 1084-1096, 2018.

[20] F. Baudart, B. Dehez, E. Matagne, D. Telteu-Nedelcu, P. Alexandre, and F. Labrique, "Torque control strategy of polyphase permanentmagnet synchronous machines with minimal controller reconfiguration under open-circuit fault of one phase," IEEE Transactions on Industrial Electronics, vol. 59, no. 6, pp. 2632-2644, 2012.

[21] G. Sala, P. Girardini, M. Mengoni, L. Zarri, A. Tani, and G. Serra, "Comparison of fault tolerant control techniques for quadruple threephase induction machines under open-circuit fault," in 2017 IEEE 11th International Symposium on Diagnostics for Electrical Machines, Power Electronics and Drives (SDEMPED), Tinos, Greece, 2017, pp. 213-219.

[22] V. F. M. B. Melo, C. B. Jacobina, and N. Rocha, "Fault tolerance performance of dual-inverter-based six-phase drive system under single-, two, and three-phase open-circuit fault operation," IET Power Electronics, vol. 11 , no. 1, pp. 212-220, 2018.

[23] V. F. M. B. Melo, C. B. Jacobina, N. Rocha, and E. R. Braga-Filho, "Fault tolerance performance of two hybrid six-phase drive systems under single-phase open-circuit fault operation," IEEE Transactions on Industry Applications, vol. 55, no. 3, pp. 2973-2983, 2019.

[24] A. Boglietti, I. R. Bojoi, S. Rubino, and M. Cossale, "Overload capability of multiphase machines under normal and open-phase fault conditions: A thermal analysis approach," IEEE Transactions on Industry Applications, vol. 56, no. 3, pp. 2560-2569, 2020.

[25] N. Bianchi, E. Fornasiero, and S. Bolognani, "Thermal analysis of a fivephase motor under faulty operations," IEEE Transactions on Industry Applications, vol. 49, no. 4, pp. 1531-1538, 2013.

[26] G. Sala, G. Valente, D. Gerada, P. Zanchetta, and C. Gerada, "Postfault operation of bearingless multisector spm machines by space vector control," IEEE Transactions on Power Electronics, vol. 35, no. 4, pp. 4168-4177, 2020.

[27] H. Zahr, M. Trabelsi, and E. Semail, "Comparison and analysis of post-fault operation modes in a five-phase pmsm considering thermal behavior," in 2018 IEEE 12th International Conference on Compatibility, Power Electronics and Power Engineering (CPE-POWERENG 2018), 2018, pp. 1-6.

[28] Yifan Zhao and T. A. Lipo, "Space vector pwm control of dual threephase induction machine using vector space decomposition," IEEE Transactions on Industry Applications, vol. 31, no. 5, pp. 1100-1109, 1995.

[29] G. Sala, M. Mengoni, G. Rizzoli, L. Zarri, and A. Tani, "Decoupled d-q axes current sharing control of multi three-phase induction machines," IEEE Transactions on Industrial Electronics, vol. 67, no. 9, pp. 71247134, 2020.

[30] M. J. Duran, I. Gonzalez-Prieto, A. Gonzalez-Prieto, and F. Barrero, "Multiphase energy conversion systems connected to microgrids with unequal power-sharing capability," IEEE Transactions on Energy Conversion, vol. 32, no. 4, pp. 1386-1395, 2017.

[31] I. Zoric, M. Jones, and E. Levi, "Arbitrary power sharing among threephase winding sets of multiphase machines," IEEE Transactions on Industrial Electronics, vol. 65, no. 2, pp. 1128-1139, 2018. 


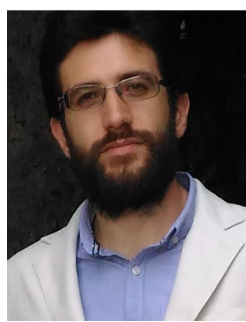

G. Sala received the B. Sc. in Power Engineering in 2012 the M. Sc. degree with honors in Electrical Engineering in 2014 and the $\mathrm{Ph}$. D. in Electrical Machines and Drives in 2018 from the University of Bologna, Italy. He worked as a researcher until 2019 in the Power Electronics, Machines and Control Group, Department of Electrical and Electronic Engineering, The University of Nottingham. Since 2019 he has been working as a researcher with the Department of Electrical, Electronic, and Information Engineering "Guglielmo Marconi" - DEI, University of Bologna, Italy, where he is currently employed as a Junior Assistant Professor, since 2020. His research interests include design, modelling and control of multiphase electrical machines, fault tolerant controls and fault diagnosis of eletric drives.

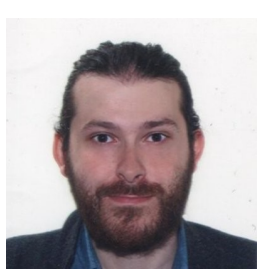

M. Mengoni (M'13) was born in Forlì, Italy. He received the M.S. (with honors) and Ph.D. degrees in electrical engineering from the University of Bologna, Bologna, Italy, in 2006 and 2010, respectively. He is currently a Senior Assistant Professor with the Department of Electric, Electronic and Information Engineering "G. Marconi", University of Bologna. His research interests include design, analysis, and control of three phase electric machines, multiphase drives, and ac/ac matrix converters.

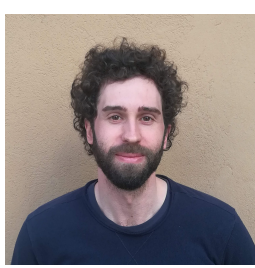

G. Rizzoli received the M.Sc and Ph.D. degree in Electrical Engineering, with honors, respectively in 2012 and 2016, from the University of Bologna, Bologna, Italy. In 2014 he was the recipient of the Best Paper Award at the IEEE International Electric Vehicle Conference 2014 (IEVC 2014). In 2015 he was a visiting student at Virginiatech (CPES), Blacksburg, Virginia, United States of America. He is currently a Junior Assistant Professor at the Department of Electrical, Electronic and Information Engineering "G. Marconi” of the University of Bologna. His research interests include the design of electrical machines, the development and control of high-efficient power converters for automotive and renewable energy applications.

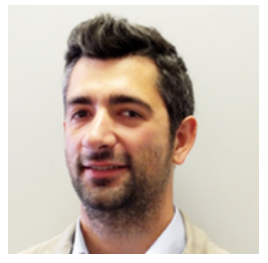

M. Degano (M'15) received his Master's degree in Electrical Engineering from the University of Trieste, Italy, in 2011, and his Ph.D. degree in Industrial Engineering from the University of Padova, Italy, in 2015. Between 2014 and 2016, he was a postdoctoral researcher at The University of Nottingham, UK, where he joined the Power Electronics, Machines and Control (PEMC) Research Group. In 2016 he was appointed Assistant Professor in Advanced Electrical Machines, at The University of Nottingham, UK. He was promoted Associate Professor in 2020. His main research focuses on electrical machines and drives for industrial, automotive, railway and aerospace applications, ranging from small to large power. He is currently the PEMC Director of Industrial Liaison leading research projects for the development of future hybrid electric aerospace platforms and electric transports.

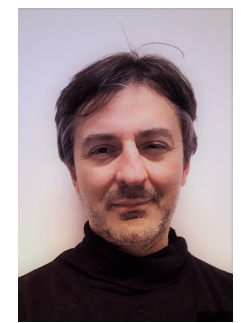

L. Zarri (M'05-SM'12) received the M.Sc. degree (Hons.) in electrical engineering and the Ph.D. degree from the University of Bologna, Bologna, Italy, in 1998 and 2007, respectively. He was a Freelance Software Programmer from 1989 to 1992 and a Plant Designer with an engineering company from 1998 to 2002. In 2003, he joined the Department of Electrical, Electronic and Information Engineering "G. Marconi", University of Bologna, as a Laboratory Engineer, where he was an Assistant Professor from 2005 to 2014, and has been an Associate Professor since 2015. He has authored or co-authored over 100 scientific papers. His current research interests include the control of power converters and electric drives. Dr. Zarri is a Senior Member of the IEEE Industry Applications Society, the IEEE Power Electronics Society, and the IEEE Industrial Electronics Society.

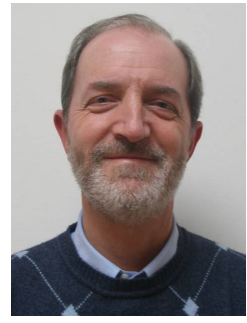

A. Tani was born in Faenza, Italy, in 1963. He received the M. Sc. in Electrical Engineering, with honors, from the University of Bologna, Bologna, Italy, in 1988. Currently he is a Full Professor of power electronics, electrical machines and drives with the Department of Electric, Electronic and Information Engineering, University of Bologna. He has authored more than 170 papers published in technical journals and Conf. proceedings. His current activities include multiphase motor drives, ac/ac induction motor drives. matrix converters, and field weakening strategies for 
TABLE III

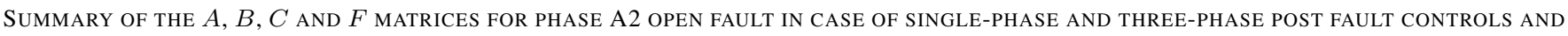
FOR SIGNIFICANT STAR CONNECTION LAYOUTS.

\begin{tabular}{|c|c|c|c|c|c|c|c|c|c|c|}
\hline Matrix $A$ (1-ph PFC) & 1.000 & 0.000 & -0.500 & -0.866 & -0.500 & 0.866 & 1.000 & 0.000 & -0.500 & -0.866 \\
\hline Matrix $B$ (1-ph PFC) & -0.500 & 0.866 & & & & & & & & \\
\hline \multirow[t]{4}{*}{ Matrix $C$ Layout $\mathrm{A}-\mathrm{B}-\mathrm{C}-\mathrm{D}^{\prime}(1-\mathrm{ph} \mathrm{PFC})$} & 1.000 & 0.000 & 0.000 & 0.000 & 0.000 & 0.000 & 0.000 & 0.000 & 0.000 & 0.000 \\
\hline & 0.000 & 1.000 & 0.000 & 0.000 & 0.000 & 0.000 & 0.000 & 0.000 & 0.000 & 0.000 \\
\hline & 0.000 & 0.000 & 0.000 & 0.000 & 0.000 & 0.000 & 1.000 & 0.000 & 0.000 & 0.000 \\
\hline & 0.000 & 0.000 & 0.000 & 0.000 & 0.000 & 0.000 & 0.000 & 1.000 & 0.000 & 0.000 \\
\hline \multirow[t]{2}{*}{ Matrix $F^{\prime}$ Layout 'A-B-C-D'(1-ph PFC) } & 0.000 & 0.000 & -0.083 & -0.144 & -0.083 & 0.144 & 0.000 & 0.000 & -0.083 & -0.144 \\
\hline & 0.000 & 0.000 & 0.144 & 0.250 & 0.144 & -0.250 & 0.000 & 0.000 & 0.144 & 0.250 \\
\hline \multirow[t]{2}{*}{ Matrix $C$ Layout 'A-C-B-D'(1-ph PFC) } & 0.500 & 1.000 & 0.000 & 0.000 & 0.000 & 0.000 & 0.500 & 0.000 & 0.000 & 0.000 \\
\hline & -0.500 & 0.000 & 0.000 & 0.000 & 0.000 & 0.000 & -0.500 & 1.000 & 0.000 & 0.000 \\
\hline \multirow[t]{2}{*}{ Matrix $F$ ' Layout 'A-C-B-D'(1-ph PFC) } & 0.063 & -0.063 & -0.063 & -0.108 & -0.063 & 0.108 & 0.063 & 0.063 & -0.063 & -0.108 \\
\hline & -0.108 & 0.108 & 0.108 & 0.188 & 0.108 & -0.188 & -0.108 & -0.108 & 0.108 & 0.188 \\
\hline Matrix $C$ Layout 'A-B-C-D'(1-ph PFC) & 2.414 & 5.828 & 0.000 & 0.000 & 0.000 & 0.000 & 2.414 & 1.000 & 0.000 & 0.000 \\
\hline \multirow[t]{2}{*}{ Matrix $F^{\prime}$ Layout 'A-B-C-D'(1-ph PFC) } & 0.083 & -0.067 & -0.056 & -0.096 & -0.056 & 0.096 & 0.083 & -0.011 & -0.056 & -0.096 \\
\hline & -0.144 & 0.116 & 0.096 & 0.167 & 0.096 & -0.167 & -0.144 & 0.020 & 0.096 & 0.167 \\
\hline \multirow[t]{3}{*}{ Matrix $A$ (3-ph PFC) } & 1.000 & 0.000 & 1.000 & 0.000 & 1.000 & 0.000 & 1.000 & 0.000 & 1.000 & 0.000 \\
\hline & 1.000 & 0.000 & -0.500 & -0.866 & -0.500 & 0.866 & 1.000 & 0.000 & -0.500 & -0.866 \\
\hline & 1.000 & 0.000 & -0.500 & 0.866 & -0.500 & -0.866 & 1.000 & 0.000 & -0.500 & 0.866 \\
\hline \multirow[t]{3}{*}{ Matrix $B$ (3-ph PFC) } & 1.000 & 0.000 & & & & & & & & \\
\hline & -0.500 & 0.866 & & & & & & & & \\
\hline & -0.500 & -0.866 & & & & & & & & \\
\hline \multirow[t]{2}{*}{ Matrix $F^{\prime}$ (3-ph PFC) } & 0.000 & 0.000 & -0.333 & 0.000 & -0.333 & 0.000 & 0.000 & 0.000 & -0.333 & 0.000 \\
\hline & 0.000 & 0.000 & 0.000 & 0.333 & 0.000 & -0.333 & 0.000 & 0.000 & 0.000 & 0.333 \\
\hline
\end{tabular}

